\title{
Domain Reconstruction and Mutation Approach to Expand Creative Design Solution Space for Practical Mechanical Systems
}

\section{Yutong Li}

China University of Petroleum Huadong

Yuxin Wang ( $\nabla$ strath_ti@hotmail.com )

China University of Petroleum Huadong

\section{Original Article}

Keywords: Creative design, domain reconstruction and mutation, complex mechanical systems, practical computerized platform

Posted Date: July 13th, 2021

DOI: https://doi.org/10.21203/rs.3.rs-698139/v1

License: (c) (1) This work is licensed under a Creative Commons Attribution 4.0 International License.

Read Full License 


\title{
Domain Reconstruction and Mutation Approach to Expand Creative Design Solution Space for Practical Mechanical Systems
}

\author{
Yu-Tong $\mathrm{Li}^{1} \quad$ - Yu-Xin Wang ${ }^{1}$
}

Received June xx, 2021; revised XX xx, 202x; accepted XX xx, 202x

(C) Chinese Mechanical Engineering Society and Springer-Verlag Berlin Heidelberg 2021

\begin{abstract}
Mechanical systems are fundamental means and carriers for expanding human physical strength. Their novelty is the key factor to determine the cost of manufacture and maintenance, service life, reliability, and other comprehensive performance of products. However, the function-based synthesis methods mainly focus on expanding the solution space in the function layer; each artifact only matches a subfunction individually. Consequently, the obtained physical structures to realize the overall function of design problems are bloated rather than concise. For overcoming this flaw, a computerized creative design system for solving non-routine design problems based on the domain synthesis theory in quotient space was proposed in our previous studies by introducing two bottom-up rule-based matching processes to replace the function-form direct mapping in the FBS model and using form-independent and solution-neutral fine granularity function units to describe the subfunctions. In this paper, the domain reconstruction and mutation processes are introduced to expand the solution space of creative design and increase the creativity of matching physical structures for mechanical systems further. The case study shows that domain reconstruction can effectively imitate human experts' creative design skills to realize the ingenious combination of mechanisms and expand the function-form mapping solution space to the creative solution space. Moreover, the domain mutation can obtain more simplified physical structures to realize the complex top-level functions with few constituent devices. Utilizing fewer and simpler constituent physical structures to meet the functional requirements synergistically determine that the physical structures of mechanical systems figured out by the platform have prominent market competitive advantages due to their structural simplicity and novelty.
\end{abstract}

Keywords: Creative design; domain reconstruction and mutation; complex mechanical systems; practical computerized platform.

Yu-Xin Wang

strath_tj@hotmail.com

College of Mechanical and Electrical Engineering, China University of Petroleum Huadong, China

\section{Introduction}

Innovation is recognized as one of the most successful strategies for capturing market share, increasing profits, and even survival [1-3]. Conceptual design is the most critical design phase to materialize an innovative product [4-6]. The novelty of conceptual design schemes is closely related to the solution space and the search number in the function-form matching process [7,8]. The abstract level of the functional descriptions has significant effects on the conceptual design solution space [9]. Using basic functional units independent of structures helps depict functions and functional relations and improve the abstract level of functional representation, thus expanding the solution space of conceptual design problems [10]. Function-based synthesis depends on the knowledge that supports its function reasoning $[11,12]$. For routine design problems [13], the main task of the computerized conceptual design system based on functional synthesis approaches is to implement the search and mapping between functions and physical structures, which results in a relatively small solution space and weakens the scheme's innovativeness [14-16]. Restricted by the complexity and uncertainty of reasoning processes and the incompleteness of reasoning knowledge [17-20], the AI-based FBS model for function reasoning mainly deals with the conceptual design of mechanical systems in series combinations [21-24] but fails to support non-routine design problems $[13,25]$. To overcome the flaws ascribed to classical function-based methods and increase the FBS model's capability to generate creative design schemes, the integrated framework $[26,27]$ to manage abstraction levels 
in problem-solving activities was proposed. Because satisfying functional requirements is regarded as the primary design task of conceptual design through solution exploration by generating a large number of alternatives, enhancing the creativeness of conceptual design results has not been adequately focused on while building up computer-based conceptual design systems.

Grammar-based design systems enable computers to generate novel solutions to design problems by exploring design alternatives according to a specific set of user-defined rules [28-32]. Viewing conceptual design as a process of coevolution of both function structures and means combinations across different levels of an abstraction hierarchy, Jin and $\mathrm{Li}$ [33] proposed a grammar-based hierarchical coevolutionary approach to support designers generating design concepts. Three genetic operations, namely, reproduction, crossover, and mutation of the genetic algorithm [34, 35], are utilized to evaluate function structures, and their corresponding solution means. Evaluating the fitness of a given function structure is a major challenge because little quantitative information is available, although two subjective terms, feasibility, and desirability, were introduced to scale the fitness in their research. Furthermore, grammar rules have magnification effects on the conceptual design solution space [36]; however, there is no extensive information on how rules change individual designs [37]. Therefore, it is difficult to determine the objective grammar rules to search the global solution space and obtain optimal innovative solutions practically and robustly [38].

Today, the proper configuration of mechanical systems and their components to meet functional requirements efficiently and can be easily manufactured and assembled is crucial to effective product design. In extant methods to expand the conceptual design solution space [7, 8, 23, 33], the relations between structural forms (or means) and functions are well-considered to generate a configuration design with desired behaviors. However, only when these desired behaviors are realized by the matched structures organically, the functional characteristics of mechanical systems can fully emerge. To our best knowledge, no literature in the field of functional synthesis deals with the behavioral continuity of matching physical structures.

Developing computerized function-based synthesis approaches to support the non-routine creative design of products is still challenging and needs to be solved by researchers [39-41]. Computer-based conceptual design systems have made significant progress in recent decades; however, computer-based creative design tools are still a technical bottleneck in solving practical engineering problems, such as complex mechanical systems [21, 25, 42]. Using solution-neutral [43] and form-independent [10] fine-granularity function units to describe the subfunctions in the FBS model [44, 45], $\mathrm{Li}$ et al. [46] proposed a bidirectional function-form matching model to enhance the creative design capability of the FBS model for solving non-routine creative design problems theoretically, mathematically, and automatically. In the model, form- and input-independent functional units, called basic operation actions, are adopted to describe the subfunctions. Two bottom-up dynamic partitioning and regrouping processes between the function and form spaces are introduced to replace function-form direct mapping in the FBS model. To make the divided subset of basic operation actions in the form space perform a well-organized and complete kinematic behavior, which can be matched by the subfunction in the function space and implemented by a physical structure in the database, a clustering approach of basic operation actions in the form space is provided [47]. Subsequently, the domain synthesis method was introduced into the function space to guarantee that the synthesized subfunctions composed and contributed by basic operation actions in the divided subsets embody continuous and orderly kinematic behaviors in time and space [48]. In this way, the generated subfunctions in the functional synthesis process can occur in time and space, both orderly and sequentially.

Further to our previous research [46-52], this paper proposes a domain reconstruction and mutation method based on quotient space to effectively expand the function-form matching solution space and enhance the innovativeness of matching physical structures.

\section{Reconstruction of Basic Operation Actions}

\subsection{Representation of Basic Operation Actions}

For mechanical systems, kinematic behaviors [53] are adopted to describe the behaviors in the FBS model. The attribute set

$$
f=\{\zeta, \gamma, \tau, S n, E n\}
$$

is utilized to describe the kinematic behavior, time, and space attributes of the basic operation actions. Where $\zeta$ represents the kinematic behavior type of a basic operation action. The kinematic behavior types can be curve motion $(C)$, circular motion $(R)$, linear motion $(L)$, and intermittent motion $(S)$. Moreover, the behavior types of the curve motion and circular motion are defined as clockwise and counterclockwise motions, respectively. 
Table 1 lists the kinematic behavior types of the basic operation actions.

Figure 1a shows a schematic diagram of a basic operation action with clockwise curve motion. $\gamma$ represents its kinematic behavior characteristics, mainly related to the velocity and direction characteristics of motion. $\tau$ represents its time attribute and describes the time required to execute the base operation action.

Table 1 List of kinematic behaviors of basic operation actions

\begin{tabular}{cccc}
\hline Types & $\begin{array}{c}\text { curve motion } \\
\text { clockwise }\end{array}$ & $\begin{array}{c}\text { curve motion } \\
\text { counterclockwise }\end{array}$ & $\begin{array}{c}\text { circle motion } \\
\text { clockwise }\end{array}$ \\
\hline Symbols & $C_{c r}$ & $C_{n c}$ & $R_{c r}$ \\
Types & $\begin{array}{c}\text { circle motion } \\
\text { counterclockwise }\end{array}$ & linear motion & $\begin{array}{c}\text { intermittent } \\
\text { motion }\end{array}$ \\
\hline Diagrams & $R_{n c}$ & $L$ & $S$ \\
\hline
\end{tabular}

$S n$ and $E n$ represent the spatial attributes of a base operation action related to its starting point and the ending point. Each basic operation action should occupy a specific space during its execution, moving from the behavior's start node to the end node. Because the path trajectory of each basic operation action in space during the conceptual design process is usually not precisely determined or defined, the relative spatial position relationships between different basic operation actions are expressed with representative frame positions as shown in Fig. $1 \mathrm{~b}$. In this figure, each horizontal or vertical grid distance represents the spatial displacement increment of a basic operation action, which is equivalent to a pulse increment of spatial displacement. Thus, the horizontal or vertical distance between different grid points in the figure represents the number of pulses spatial displacement that a basic operation action needs to span in a specific period. For instance, the function of "clamping" is implemented by two basic operation actions $b_{m}$ and $b_{n}$. Here, $b_{m}$ is the basic operation action from $(2,2)$ to $(2,1)$ with linear motion, and $b_{n}$ is the basic operation action from $(2,0)$ to $(2,1)$ with linear motion. Therefore, the representative frame positions in Fig. 1b are only used to describe the spatial position characteristics of the movement behaviors of basic operation actions rather than to describe the actual specific spatial displacement of basic operation actions. The reason to do so is that the kinematic output of a mechanism is closely related to its dimensions, which are uncertain in the conceptual design stage.

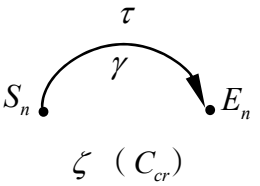

(a) curve motion

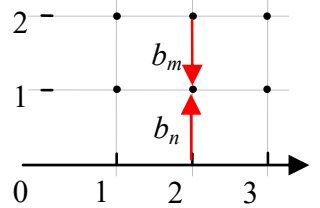

(b) frame positions
Figure 1 Attributes and representative frame positions of basic operation actions

\subsection{Reconstruction Rules of Basic Operation Actions}

Rule 1: Two basic operation actions of linear motion occurring in the time order with the same motion direction and acting line, and continuous spatial positions, can be composed.

To facilitate computer calculation, this reconstruction rule is expressed in quotient space [54] with the quotient attributes of basic operation actions as follows: if two basic operation actions $b_{\alpha}, b_{\beta} \in[x]_{i}$ satisfy the order relation in quotient space $b_{\alpha}<b_{\beta}$ (or $b_{\beta}<b_{\alpha}$ ), and $b_{\alpha} \cdot \zeta=b_{\beta} \cdot \zeta=L, b_{\alpha} \cdot E n=b_{\beta} \cdot S n$ (or $b_{\alpha} \cdot S n=b_{\beta} \cdot E n$ ), $\vec{b}_{\alpha} \cdot \vec{b}_{\beta}=0$, then they can be synthesized as a basic operation action. The synthesis process is represented by $b_{\alpha} \oplus b_{\beta}=b_{\gamma}$. Where $b_{\gamma} \cdot \zeta=L, \quad b_{\gamma} \cdot \tau=b_{\alpha} \cdot \tau+b_{\beta} \cdot \tau, \quad b_{\gamma} \cdot S n=b_{\alpha} \cdot S n \quad$ (or $\left.b_{\beta} \cdot S n\right), b_{\gamma} \cdot E n=b_{\beta} \cdot E n$ (or $b_{\alpha} \cdot E n$ ). Note: here, $b_{\alpha} \cdot \zeta$ represents the behavior type of basic operation action $b_{\alpha}$; $L$ represents the linear motion or other types of kinematic behaviors; $\vec{b}_{\alpha}$ represents the configuration vector of basic operation action $b_{\alpha}$, that is, the vector pointing from point $b_{\alpha} \cdot S n$ to point $b_{\alpha} \cdot E n$; and $\oplus$ represents the synthesis calculation. Figure $2 \mathrm{a}$ shows the synthesis process.

Rule 2: Two basic operation actions of linear motion coinciding with the same acting line, but with opposite directions, can be composed.

The mathematical expression of this rule in quotient space is as follows: if two basic operation actions $b_{\alpha}, b_{\beta} \in[x]_{i}$ satisfy the order relation $b_{\alpha}<b_{k}$ and $b_{\beta}<b_{k}$ (or $b_{k}<b_{\alpha}$ and $b_{k}<b_{\beta}$ ), and $b_{\alpha} \cdot \tau=b_{\beta} \cdot \tau$, $b_{\alpha} \cdot \zeta=b_{\beta} \cdot \zeta=L, \vec{b}_{\alpha} \cdot \vec{b}_{\beta}=0, b_{\alpha} \cdot E n=b_{\beta} \cdot E n\left(\right.$ or $\left.b_{\alpha} \cdot S n=b_{\beta} \cdot S n\right)$, then they can be synthesized as a basic operation action. The synthesis process can be represented as $b_{\alpha} \oplus b_{\beta}=b_{\gamma}$. Where $b_{\gamma} \cdot \zeta=L, b_{\gamma} \cdot \tau=b_{\alpha} \cdot \tau=b_{\beta} \cdot \tau, b_{\gamma} \cdot S n=b_{\alpha} \cdot S n$, $b_{\gamma} \cdot E n=b_{\beta} \cdot E n \quad\left(\right.$ or $\left.b_{\gamma} \cdot S n=b_{\beta} \cdot S n, b_{\gamma} \cdot E n=b_{\alpha} \cdot E n\right)$. The synthesis process is shown in Fig. $2 b$.

Since the actual spatial displacements of $b_{\alpha}$ and $b_{\beta}$ are not equal to each other, when the actual spatial displacement of $b_{\alpha}$ is greater than one of $b_{\beta}$, the representative displacement of $b_{\gamma}$ is shown as the right upper vector in Fig. $2 b$. That is, the representative 
displacement of the combined $b_{\gamma}$ passes through the point $(2,1)$ from the point $(1,1)$ and continues to move towards with less than the value of the representative displacement of $b_{\beta}$, and reaches the point $\left(2+\left.\Delta\right|_{\Delta<1}, 1\right)$. Otherwise, the representative displacement of $b_{\gamma}$ is shown as the right lower vector in Fig. 2b.

Rule 3: Two basic operation actions of curve motion occurring clockwise in the time order and satisfying a specific position relation can be composed.

The expression is as follows: if two basic operation actions $b_{\alpha}, b_{\beta} \in[x]_{i}$ satisfy the order relation $b_{\alpha}<b_{\beta}$ (or $b_{\beta}<b_{\alpha}$ ) and $b_{\alpha} \cdot \zeta=b_{\beta} \cdot \zeta=C_{c r}$ (or $b_{\alpha} \cdot \zeta=b_{\beta} \cdot \zeta=R_{c r}$ ), $90^{\circ}>\theta_{\alpha \beta}>0, d_{\alpha \beta}<0, b_{\alpha} \cdot E n=b_{\beta} \cdot S n \quad\left(\right.$ or $\left.b_{\alpha} \cdot S n=b_{\beta} \cdot E n\right)$, then they can be synthesized as a basic operation action. The synthesis process is represented by $b_{\alpha} \oplus b_{\beta}=b_{\gamma}$. Where $b_{\gamma} \cdot \zeta=C_{c r} \quad\left(\right.$ or $\left.b_{\gamma} \cdot \zeta=R_{c r}\right), b_{\gamma} \cdot \tau=b_{\alpha} \cdot \tau+b_{\beta} \cdot \tau$, $b_{\gamma} \cdot S n=b_{\alpha} \cdot S n\left(\right.$ or $\left.b_{\beta} \cdot S n\right)$, and $b_{\gamma} \cdot E n=b_{\beta} \cdot E n\left(\right.$ or $\left.b_{\alpha} \cdot E n\right)$. Here, $\theta_{\alpha \beta}=\arccos \left(\vec{b}_{\alpha} \cdot \vec{b}_{\beta} /\left|b_{\alpha}\right| \cdot\left|b_{\beta}\right|\right)$ is the angle of $b_{\alpha}$ and $\vec{b}_{\beta} ; d_{\alpha \beta}$ represents the rotating direction of vector $\vec{b}_{\beta}$ related to vector $\vec{b}_{\alpha}$. Here, $d_{\alpha \beta}=\arccos \left(\vec{b}_{\beta} \times \vec{b}_{\alpha} /\left|\vec{b}_{\beta} \times \vec{b}_{\alpha}\right|\right)$. The composition process is shown in Fig. 2c.

Rule 4: Two basic operation actions of curve motion occurring counterclockwise in the time order and meeting a particular position relation can be composed.

The expression is as follows: if two basic operation actions $b_{\alpha}, b_{\beta} \in[x]_{i}$ satisfy the order relation $b_{\alpha}<b_{\beta}$ (or $\left.b_{\beta}<b_{\alpha}\right)$ and $b_{\alpha} \cdot \zeta=b_{\beta} \cdot \zeta=C_{n c}\left(\right.$ or $\left.b_{\alpha} \cdot \zeta=b_{\beta} \cdot \zeta=R_{n c}\right)$, $90^{\circ}>\theta_{\alpha \beta}>0, d_{\alpha \beta}>0, b_{\alpha} \cdot E n=b_{\beta} \cdot S n \quad\left(\right.$ or $\left.b_{\alpha} \cdot S n=b_{\beta} \cdot E n\right)$, then they can be synthesized as a basic operation action. The synthesis process is represented by $b_{\alpha} \oplus b_{\beta}=b_{\gamma}$. Where $b_{\gamma} \cdot \zeta=C_{n c} \quad\left(\right.$ or $\left.\quad b_{\gamma} \cdot \zeta=R_{n c}\right), \quad b_{\gamma} \cdot S n=b_{\alpha} \cdot S n \quad$ (or $\left.b_{\beta} \cdot S n\right), b_{\gamma} \cdot E n=b_{\beta} \cdot E n$ (or $b_{\alpha} \cdot E n$ ), and $b_{\gamma} \cdot \tau=b_{\alpha} \cdot \tau+b_{\beta} \cdot \tau$. The synthesis process is shown in Fig. 2 d.

Rule 5: Two basic operation actions, one in clockwise curve motion and another in counterclockwise curve motion co-occurring and satisfying a particular position relation, can be combined.

The expression is as follows: if two basic operation actions $b_{\alpha}, b_{\beta} \in[x]_{i}$ satisfy the order relation $b_{\alpha}<b_{k}$ and $b_{\beta}<b_{k}$ (or $b_{k}<b_{\alpha}$ and $b_{k}<b_{\beta}$ ) and $b_{\alpha} \cdot \zeta=C_{c r}$, $b_{\beta} \cdot \zeta=C_{n c}\left(\right.$ or $\left.b_{\alpha} \cdot \zeta=R_{c r}, b_{\beta} \cdot \zeta=R_{n c}\right), 180^{\circ}>\theta_{\alpha \beta}>90^{\circ}$, $b_{\alpha} \cdot \tau=b_{\beta} \cdot \tau, b_{\alpha} \cdot E n=b_{\beta} \cdot E n$ (or $\left.b_{\alpha} \cdot S n=b_{\beta} \cdot S n\right)$, then their synthesis process can be represented as $b_{\alpha} \oplus b_{\beta}=b_{\gamma}$. Where $b_{\gamma} \cdot \zeta=C_{c r}\left(\right.$ or $\left.b_{\gamma} \cdot \zeta=R_{c r}\right), \quad b_{\gamma} \cdot \tau=b_{\alpha} \cdot \tau=b_{\beta} \cdot \tau$, $b_{\gamma} \cdot S n=b_{\alpha} \cdot S n$, and $b_{\gamma} \cdot E n=b_{\beta} \cdot S n \quad$ (or $b_{\gamma} \cdot S n=b_{\beta} \cdot E n$, $\left.b_{\gamma} \cdot E n=b_{\alpha} \cdot E n\right)$. The composition process is shown in Fig. 2e. The formation reason for the representative displacement of the combined $b_{\gamma}$ in such form as shown in Fig. 2e is similar to Fig. 2 b.

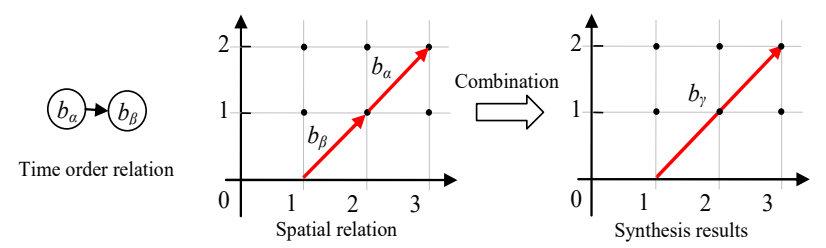

(a) Two linear motions with the same direction
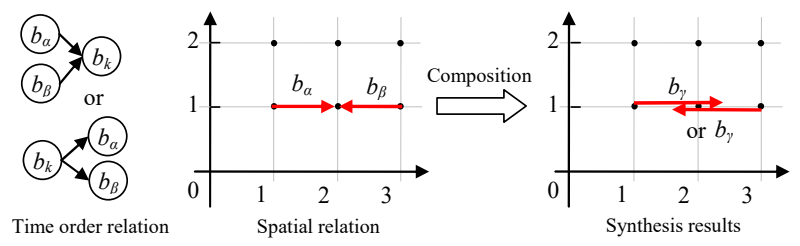

(b) Two linear motions with the opposite directions

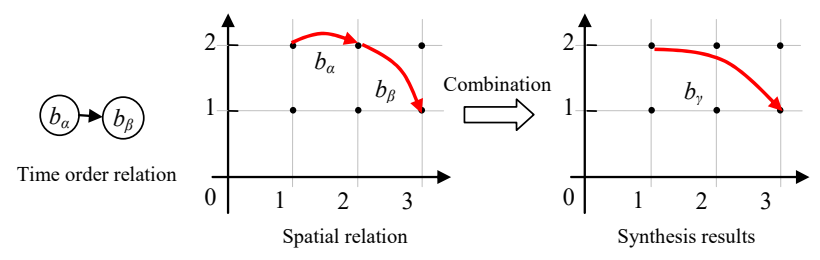

(c) Two curve motions occur in order and clockwise

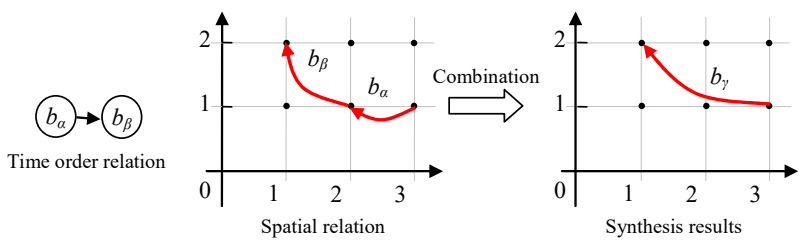

(d) Two curve motions occur in order and counterclockwise
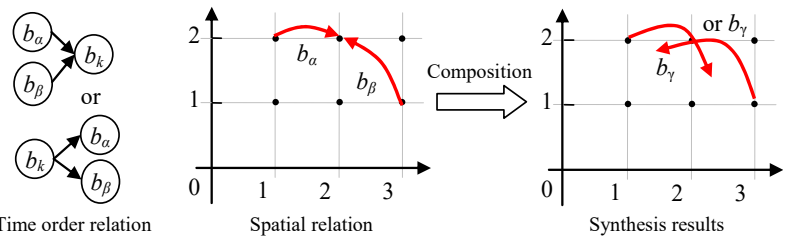

(e) Two curve motions occur at the same time in opposite directions
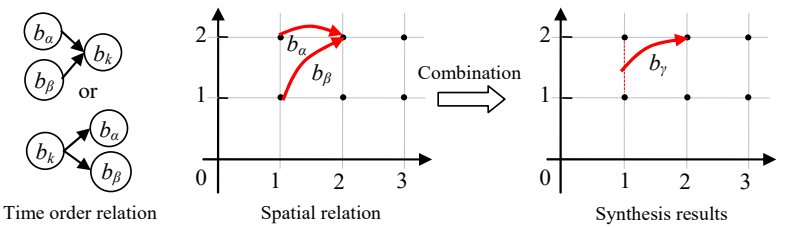

(e) Two basic operation actions occur at the same time with the same ending point

Figure 2 Combination of two basic operation actions

Rule 6: Two basic operation actions that co-occur with the same ending point can be composed.

Its mathematical expression is as follows: if two basic operation actions $b_{\alpha}, b_{\beta} \in[x]_{i}$ satisfy the order relation $b_{\alpha}<b_{k}$ and $b_{\beta}<b_{k}$ (or $b_{k}<b_{\alpha}$ and $b_{k}<b_{\beta}$ ), and $b_{\alpha} \cdot \zeta=I, b_{\beta} \cdot \zeta=I, 180^{\circ}>\theta_{\alpha \beta}>0, b_{\alpha} \cdot \tau=b_{\beta} \cdot \tau$, $b_{\alpha} \cdot E n=b_{\beta} \cdot E n \quad\left(\right.$ or $\left.b_{\alpha} \cdot S n=b_{\beta} \cdot S n\right)$, then they can be synthesized as a basic operation action, and the synthesis 
process can be represented with $b_{\alpha} \oplus b_{\beta}=b_{\gamma}$. Where $b_{\gamma} \cdot \zeta=I, \quad b_{\gamma} \cdot \tau=b_{\alpha} \cdot \tau=b_{\beta} \cdot \tau, b_{\gamma} \cdot E n=b_{\alpha} \cdot E n \quad\left(\right.$ or $b_{\gamma} \cdot S n$ $\left.=b_{\beta} \cdot S n\right)$, and $b_{\gamma} \cdot E n=M n$. Here, $I$ denotes the type of kinematic behavior. It can be $R_{c r}, R_{n c}, C_{c r}, C_{n c}$. Mn is the midpoint of the starting points of the two basic operation actions. The synthesis process is shown in Fig. $2 \mathrm{f}$.

Rule 7: For two basic operations of intermittent motion at the same node, if they occur in the time order continuously, they can be combined as a basic operation action with intermittent kinematic behavior whose duration time is the sum of the two operation actions. If two basic operation actions of intermittent motion co-occur with the same node, they can be synthesized as a basic operation action whose duration time equals the duration of one.

Based on the above seven reconstruction rules, the basic operation actions in the functional space can be reconstructed. For a subfunction with more than two basic operation actions, two can be synthesized into new kinematic behavior according to the above seven rules. This synthesized kinematic behavior is taken as a new basic operation action to synthesize with another basic operation action and form a new synthesized kinematic behavior. Until the synthesized kinematic behavior or subfunction meets the granularity requirements and matches the physical structure stored in the database [52].

To make the synthesized subfunctions contributed by all basic operation actions in the synthesized subsets to form united kinematic behaviors and take place in time and space continuously and orderly, a domain synthesis approach was proposed by Li and Wang [47]. During the domain synthesis process, the synthesized space related to the granularity of basic operation actions is divided into two quotient spaces: the topological structure synthesized space and the functional attribute synthesized space, corresponding to the temporal order equivalence relations and the spatial position equivalence relations, respectively. The temporal order relations and spatial position relations among the synthesized subsets and their constituent basic operation actions are guaranteed by temporal order-based partitioning rules and spatial position-based partitioning rules, respectively. The domain synthesized space is the intersection of the topological structure synthesized space and the functional attribute synthesized space. Therefore, the kinematic behaviors contributed by all elements in each synthesized subset can satisfy the time-continuity and spatial positions-continuity requirements. The synthesized subfunctions obtained in the domain synthesis process can realize a given top-level function organically and cooperatively. For this type of characteristic, the consistency between the synthesized subfunctions and the top-level function will not be discussed here while carrying out the domain reconstruction and mutation.

\section{Mutation of Basic Operation Actions}

\subsection{Mutation Rules}

The mutation operation in the genetic algorithm for optimization problems [34, 35] refers to the random changing of the value ranges of variables to expand the value spaces of optimization variables, prevent the local convergence of optimization problems, and get globally optimal results. However, here, the mutation is the operation of changing the kinematic types of basic operation actions in a subset to different types according to the mutation rules, without changing the kinematic behaviors of the subset, thereby obtaining creative conceptual design results. Similar to the principles described in Section 2, single or multiple basic operation actions in the subset can be mutated to other types of basic operation actions. Then, innovation occurs concerning the original schemes under the condition that the subset's functions remain unchanged. The mutation operation rules are as follows:

Rule 1: Two basic operation actions in linear motion reciprocating at the same positions can be transformed into two reciprocating basic operation actions in curve motion.

The mutation operation can be expressed as follows: if two basic operation actions $b_{\alpha}, b_{\beta} \in[x]_{i}$ satisfy the order relation $b_{\alpha}<b_{\beta}$, and $b_{\alpha} \cdot \zeta=b_{\beta} \cdot \zeta=L, b_{\alpha} \cdot \tau=b_{\beta} \cdot \tau$, $b_{\alpha} \cdot S n=b_{\beta} \cdot E n \quad\left(\right.$ or $\left.b_{\alpha} \cdot E n=b_{\beta} \cdot S n\right)$, then the mutation operation, as shown in Fig. 3a, is represented as $b_{\alpha} \stackrel{\mathrm{v}}{\longrightarrow} b_{\alpha}^{\prime}, b_{\beta} \stackrel{\mathrm{v}}{\longrightarrow} b_{\beta}^{\prime}$. Where " $\stackrel{\mathrm{V}}{\longrightarrow}$ " is the mutation calculation, $b_{\alpha}^{\prime} \cdot E n=b_{\beta}^{\prime} \cdot S n=b_{\alpha} \cdot E n, b_{\alpha}^{\prime} \cdot S n=b_{\beta}^{\prime} \cdot E n=b_{\alpha} \cdot S n$, $b_{\alpha}^{\prime} \cdot \tau=b_{\beta}^{\prime} \cdot \tau=b_{\alpha} \cdot \tau, b_{\alpha}^{\prime} \cdot \zeta=C_{c r}$, and $b_{\beta}^{\prime} \cdot \zeta=C_{n c}$. The mutation operation is invertible, that is, two basic operation actions in curve motion can also be transformed into two in linear motion.

Rule 2: Two basic operation actions in linear motion, reciprocating at the same positions and resting at the middle position, can be transformed into two basic operation actions in curve motion.

The mutation operation can be expressed as follows: if three basic operation actions $b_{\alpha}, b_{\beta}, b_{\chi} \in[x]_{i}$ satisfy the order relation $b_{\alpha}<b_{\chi}<b_{\beta}$, and if $b_{\alpha} \cdot \zeta=b_{\beta} \cdot \zeta=L$, $b_{\alpha} \cdot S n=b_{\beta} \cdot E n \quad\left(\right.$ or $\left.\quad b_{\alpha} \cdot E n=b_{\beta} \cdot S n \quad\right), \quad b_{\alpha} \cdot \tau=b_{\beta} \cdot \tau$, $b_{\chi} \cdot \zeta=S$, then the mutation operation, as shown in Fig. 
$3 \mathrm{~b}$, can be represented as $b_{\alpha} \stackrel{\mathrm{v}}{\longrightarrow} b_{\alpha}^{\prime}, b_{\beta} \stackrel{\mathrm{v}}{\longrightarrow} b_{\beta}^{\prime}$. Where the mutation calculation is $b_{\alpha}^{\prime} \cdot \zeta=C_{c r}, b_{\beta}^{\prime} \cdot \zeta=C_{n c}$, $b_{\alpha}^{\prime} \cdot \tau=b_{\beta}^{\prime} \cdot \tau=b_{\alpha} \cdot \tau, b_{\alpha}^{\prime} \cdot E n=b_{\beta}^{\prime} \cdot S n=b_{\alpha} \cdot E n$ and $b_{\alpha}^{\prime} \cdot S n=b_{\beta}^{\prime} \cdot E n$ $=b_{\alpha} \cdot S n$. The mutation process is invertible.

Rule 3: A basic operation action in the middle of two basic operation actions with the same kinematic behavior can be mutated into another kinematic behavior.

The expression is as follows: if three basic operation actions $b_{\alpha}, b_{\beta}, b_{\chi} \in[x]_{i}$ satisfy the order relation $b_{\alpha}<b_{\chi}<b_{\beta}$, and $b_{\alpha} \cdot \zeta=b_{\beta} \cdot \zeta=I, b_{\chi} \cdot S n=b_{\alpha} \cdot E n, b_{\chi} \cdot E n=b_{\beta} \cdot S n, b_{\chi} \cdot \zeta=J$ and $90^{\circ}>\theta_{\alpha \beta}>0$, then the mutation process can be represented as $b_{\chi} \stackrel{\mathrm{v}}{\longrightarrow} b_{\chi}^{\prime}$. Where $b_{\chi}^{\prime} \cdot \tau=b_{\chi} \cdot \tau, b_{\chi}^{\prime} \cdot \zeta=K$, $b_{\chi}^{\prime} \cdot S n=b_{\chi} \cdot S n$, and $b_{\chi}^{\prime} \cdot E n=b_{\chi} \cdot E n$. The variables $I, J, K \in$ $\left\{L, R_{c r}, R_{n c}, C_{c r}, C_{n c}\right\}$ are the types of kinematic behaviors of the basic operation actions. All possible corresponding kinematic behaviors for line and curve motions are listed in Table 1. The mutation operation for a basic operation action of clockwise curve motion in the middle of two basic operation actions with the same kinematic behavior to transform into linear motion is shown in Fig. 3c.

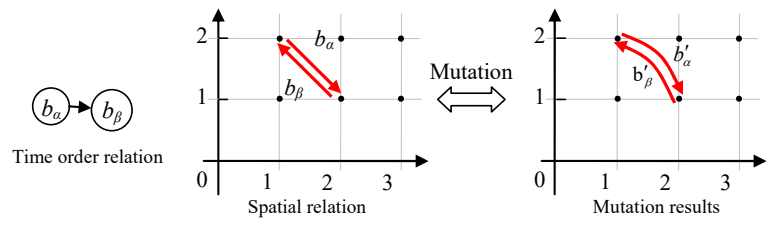

(a) Two reciprocating linear motions are transformed into two reciprocating curve motions

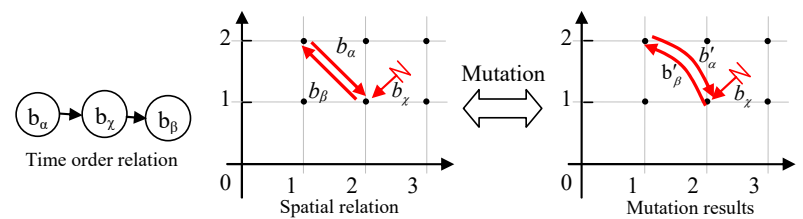

(b) Two linear motions with rest motion at the middle position

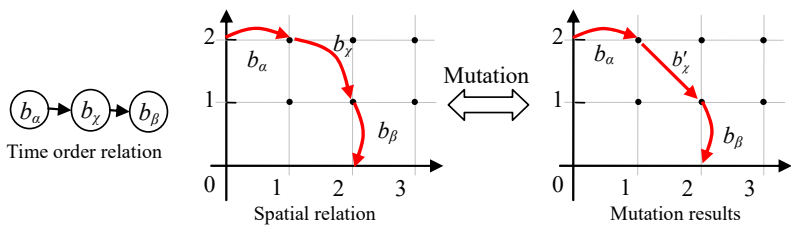

(c) A basic operation action in the middle of two curve motions

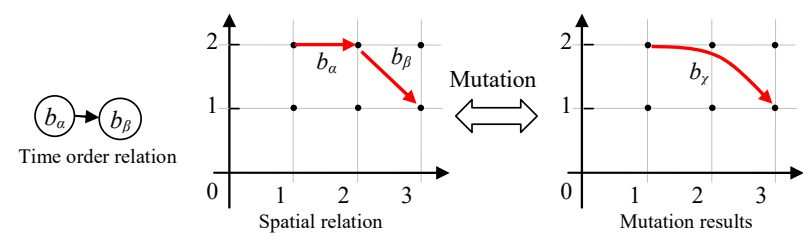

(d) Two linear motions are transformed into one curve motion

Figure 3 Mutation of basic operation actions

Rule 4: Two basic operation actions in linear motion occurring continuously and satisfying a particular position relation can be mutated into a basic operation action in curve motion.

The expression is as follows: if two basic operation actions $b_{\alpha}, b_{\beta} \in[x]_{i}$ satisfy the order relation $b_{\alpha}<b_{\beta}$, and $b_{\alpha} \cdot \zeta=b_{\beta} \cdot \zeta=L, 90^{\circ}>\theta_{\alpha \beta}>0$, and $b_{\beta} \cdot S n=b_{\alpha} \cdot E n$, when $d_{\alpha \beta}<0$, the mutation operation can be represented as $b_{\alpha} \oplus b_{\beta} \stackrel{\mathrm{v}}{\longrightarrow} b_{\chi} \quad$, and $b_{\chi} \cdot \zeta=C_{c r}, \quad b_{\chi} \cdot \tau=b_{\alpha} \cdot \tau+b_{\beta} \cdot \tau$, $b_{\chi} \cdot S n=b_{\alpha} \cdot S n$ and $b_{\chi} \cdot E n=b_{\beta} \cdot E n$; when $d_{\alpha \beta}>0$, the mutation operation is $b_{\alpha} \oplus b_{\beta} \stackrel{\mathrm{v}}{\longrightarrow} b_{\chi}^{\prime}$, and $b_{\chi}^{\prime} \cdot \zeta=C_{n c}$, $b_{\chi}^{\prime} \cdot \tau=b_{\alpha} \cdot \tau+b_{\beta} \cdot \tau, b_{\chi}^{\prime} \cdot S n=b_{\alpha} \cdot S n$, and $b_{\chi}^{\prime} \cdot E n=b_{\beta} \cdot E n$. The mutation operation is shown in Fig. $3 \mathrm{~d}$.

Suppose that the basic operation actions in the subset obtained through the domain synthesis process meet one of the above four mutation operation conditions. These basic operation actions are then subjected to mutation operation. It should be emphasized that the mutation operation does not change the subsets' total functional characteristics, but expands the functional solution space.

\subsection{Case Study for Mutation Operation}

Here, we consider the functional requirements for a clamp to illustrate the domain reconstruction and mutation operation. The functional requirements of the clamp are shown in Fig. 4. Because basic operation actions $b_{1}$ and $b_{3}$ satisfy the second reconstruction rule in Section 2, i.e., $b_{1}$ and $b_{3}$ of linear motion coinciding with the same acting line, but with opposite directions, they can be composed into one basic operation action $b_{c 1}$. Similarly, $b_{2}$ and $b_{4}$ can be formed as a basic operation action $b_{c 2}$. The synthesized results are shown in Fig. 5a. Consequently, the number of basic operation actions required to implement the clamp's top-level function is reduced to two. Moreover, a new working principle is formed, that is, one paw is fixed, and the other moves close to or far away.
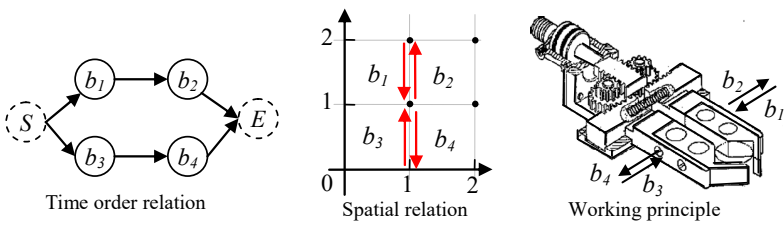

Figure 4 Functional requirements for a clamp and basic operation actions

Because basic operation actions $b_{1}$ and $b_{2}$ satisfy the first mutation rule, they can be mutated into basic operation actions in curve motion, denoted as $b_{1}^{\prime}$ and $b_{2}^{\prime}$. Similarly, $b_{3}$ and $b_{4}$ can also be mutated into basic operation actions in curve motion, denoted as $b_{3}^{\prime}$ and $b_{4}^{\prime}$. Furthermore, because basic operation actions $b_{1}^{\prime}$ and $b_{4}^{\prime}$ satisfy the third reconstruction rule, they can be composed 
as a basic operation action $b_{c 1}^{\prime}$. Similarly, $b_{3}^{\prime}$ and $b_{2}^{\prime}$ can be composed as a basic operation action $b_{c 2}^{\prime}$. The mutation results are shown in Fig. $5 b$.

It can be found that both the reconstruction and mutation of basic operation actions in the functional space can lead to new functional schemes (or functional working principles). They expand the functional solution space significantly and generate novel functional working principles. As shown in Fig. 5b, the simplified functional working principles lead to matched constituent physical structures with simple and optimal configurations to meet the functional requirements.
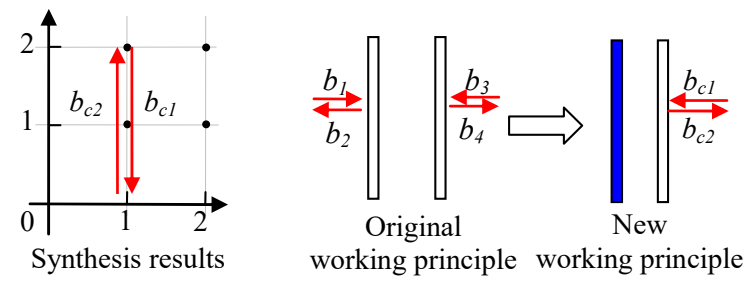

(a) Reconstruction

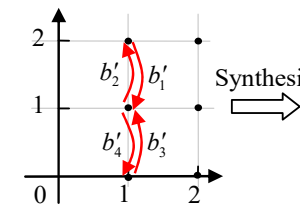

Mutation results

(b) Mutation

Figure 5 Domain reconstruction and mutation for clamp's functional requirements

\section{Creative Design Platform for Practical Mechanical Systems}

\subsection{Novelty for Synthesis of Mechanical Systems}

Mechanical systems are fundamental means and carriers for expanding human physical strength. The innovative nature of the structures that comprise them is a critical factor determining product manufacturing and maintenance costs, service life, reliability, and other market competitive advantages. Their high level of innovation is of great value for improving and enhancing human material and spiritual life. With the development of computer control technology, modern mechanical systems have become highly integrated systems of machinery and computer control systems [2]. Flexibility, controllability, and collaboration are the dominant characteristics of their kinematic behaviors; multiple degrees of freedom, motion combination, and integration are their structural features, and the mechanisms are the implementers of their kinematic behaviors. Limited by the kinematic behaviors of a single common mechanism, a complex mechanical system, such as textile machinery, usually comprises several common mechanisms with certain connection relationships to implement complex kinematic behaviors in an orderly and concerted manner. Therefore, the combined mechanism is a dominant pattern that constitutes the kinematic schemes of modern mechanical systems.

AI-based function reasoning techniques can efficiently deal with the synthesis of combined mechanisms in series connections for input/output functional requirements [7, $11,16]$. However, they cannot be applied to deal with the synthesis of combined mechanisms in parallel and compound connections because of a lack of direct, indirect, or even implicit knowledge to support the reasoning process of mechanism combination from constituent mechanisms to their combination. This is because when a complex mechanism is combined in parallel or compound connections, its kinematic behaviors will mutate [55], which is by no means equivalent to the simple combination of kinematic behaviors of each constituent mechanism.

Conversely, for human experts, conceiving kinematic schemes for complex mechanical systems is never a simple assembly process but rather entails weighing the design requirements, scheme complexity [56] and cost, coordination between different subfunctions, design constraints, and the characteristics of each constituent mechanism, including its auxiliary functions. Only the most optimal ingenious combinations of common mechanisms meeting all design requirements can be selected as candidates for design problems. If this kind of ingenious mechanism combination by human experts can be simulated and implemented with a computerized approach, a computer-based conceptual design tool for combined mechanisms can generate more innovative conceptual design schemes whose innovation level will not be inferior to human experts.

\subsection{Creative Design Platform for Practical Mechanical Systems}

Building a computerized creative design system and platform to solve non-routine creative design problems for practical complex mechanical systems is a challenge for international scholars $[8,15,25]$. It has great merit and significance in improving the creativity and market competitiveness of mechanical products. In the past two decades, we have struggled to solve the above puzzle for deriving more innovative mechanical systems that are not 
inferior to the level of innovation of human experts with the aid of computers' powerful computation capacity, focusing on the synthesis of combined mechanisms in parallel and composite connections mainly.

Doubtlessly, the system constituted with fewer and simpler building blocks, i.e., mechanisms for mechanical systems, to meet the functional requirements of design problems has prominent market competitive advantages in cost, life, reliability, and other performances. Because each building block in the FBS model only embodies one individual subfunction independently, the obtained physical structures to realize the overall function are quite incompact and bloated [15, 25]. For obtaining embodied physical structures to meet function requirements with fewer and simpler building blocks, a computerized non-routine creative design platform for mechanical systems, including light industry machinery, printing machinery, packaging machinery, automatic machinery, textile machinery, and others, is constructed.

The platform consists of three parts: a creative design unit, virtual simulation unit, and supporting database. The creative design unit includes the functional decomposition module [49], rule-based generative module [51], domain synthesis module [48], and domain reconstruction and mutation module based on the method presented in this paper. Here, the functional-structure obtained in the functional decomposition module in the FBS model, as shown in Fig. 6a, is only used as an initial function scheme for the reconstruction and mutation of basic operation actions. The method of obtaining the initial function scheme shown in Fig. 6a can also be the FBS-based systematic method [7,10] or other methods, such as the TRIZ-based approach [6].

For evaluating the physical structures generated in the creative conceptual design process, a virtual simulation tool supporting automatic simulation of the physical structures represented by symbolic schemes was provided by $\mathrm{Li}$ and Wang [50]. It contains the recognition of symbolic schemes, motion analysis, solid structure modeling, layout design, 3D virtual simulation, and other automated processes related to automatic virtual simulation of generated innovative schemes.

The computerized platform to implement functional innovation is based on two processes: the functional domain synthesis process and the domain reconstruction and mutation process. The domain synthesis process is a bottom-up functional synthesis process of the function layer. The function domain is clustered and divided into many functional schemes and hierarchies based on dynamic partition rules constructed related to the temporal and spatial relations among basic operation actions. Different partitions will lead to different sub-functions and functional structures for the same conceptual design problem. So it has a solid capability to introduce new design variables into the functional synthesis process and provides a great many functional hierarchies. The domain reconstruction and mutation processes introduced in this paper further expand the functional and physical solution space to the creative design space [13].

The innovation of the constituted physical structures is realized by making the multiple behaviors of each building block participate in function-form matching and enlarging the number of basic operation actions in the matching subfunctions. Thus, more simplified mechanical systems with few constituent mechanisms can embody the top-level functional requirements of the design problems. The functional creativity and the innovation of constituted physical structures are illustrated in Section 5.

\subsection{Database for Creative Design Platform}

Establishing a database is essential for constructing an automated creative conceptual design platform for practical complex mechanical systems. Here, MySQL is utilized to build up a database for practical mechanical systems. It includes three parts: the library of basic operation actions, the library of functions, and the library of physical structures for mechanical systems. In the library of physical structures, the common constituent structures from printing machinery, packaging machinery, automatic machinery, textile machinery, and other types of machinery are collected.

The basic operation actions in the library of basic operation actions are defined by their source, behavior, and quality attributes [52]. The source attributes indicate the origins of basic operation actions from a common basic mechanism in the database and their connecting relations. It contains essential information for partitioning the basic operation action set and matching the physical structures. The most important aspects of kinematic behaviors are the behavior module, behavior characteristics, and generalized behavior of the input and output of a basic operation action. The quality attribute is a qualitative description of the output behavior of basic operation actions and serves as a criterion for judging the effectiveness and selection of the creative design schemes.

The library consists of 305 basic operation actions from mechanical, pneumatic and hydraulic, sensing, and control systems. A total of 319 structural functions are stored in the library of functions, including 215 mechanical systems, 83 pneumatic and hydraulic systems, and 21 sensing and 
control systems. The structural function is defined by a set of basic operational actions that comprise kinematic behaviors. The function library stores the IDs related to the set of basic operation actions. The details of these basic operation actions can be read out by indexing the ID. There are 221 physical structures of electromechanical and hydraulic integration systems stored in the structure library. The executing function of a physical structure in the database is defined with basic operation actions and additional information. Each executing function includes a primary function and several auxiliary functions: the multi-function kinematic behaviors shown in Table 2 for Gear. The additional information mainly consists of the input and output node information of the source structure functions, which provide spatial location information and link information for the basic operation actions of the constituent structures that match the subfunctions obtained through functional domain reconstruction and mutation.

Table 2 Typical building block (a basic mechanism) stored in the

\begin{tabular}{|c|c|c|c|c|c|c|c|}
\hline Name & ID & 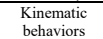 & BOA 1 & $\mathrm{BOA} 2$ & $\mathrm{BOA} 3$ & $\begin{array}{c}\text { Input } \\
\text { panel points }\end{array}$ & $\begin{array}{c}\text { Output panel } \\
\text { points }\end{array}$ \\
\hline $\begin{array}{l}\text { Epicycle } \\
\text { gear train 2 }\end{array}$ & GEAR_08_02 & \multirow{3}{*}{$\begin{array}{l}\text { Primary } \\
\text { function } \\
\text { Auxiliary } \\
\text { function 1 } \\
\text { Auxiliary } \\
\text { function 2 }\end{array}$} & OP_CM_03 & OP_CM_01 & OP_CCM_01 & $1 \& 3$ & 4 \\
\hline \multicolumn{2}{|c|}{ Diagram } & & OP_CM_03 & OP_CM_01 & OP_CM_25 & $1 \& 3$ & 2 \\
\hline \multirow[t]{2}{*}{$\bar{\beta}$} & \multirow{5}{*}{$4^{\frac{1}{1}}$} & & Null & Null & Null & Null & Null \\
\hline & & \multirow{4}{*}{$\begin{array}{c}\text { Executing } \\
\text { function } \\
\text { representation }\end{array}$} & \multicolumn{4}{|l|}{$\begin{array}{l}\text { Primary } \\
\text { Function }\end{array} \rightarrow$} & \multirow{4}{*}{$\begin{array}{l}\text { Auxiliary } \\
\text { Function } \\
\begin{array}{l}\text { Number of } \mathrm{BOA} A \\
\text { in the database }\end{array}\end{array}$} \\
\hline \multirow{3}{*}{ 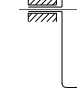 } & & & & $\mathrm{OP}_{-} \mathrm{CM}$ & & OP_CM_03 & \\
\hline & & & & - $P_{-} \mathrm{CM}$ & & OP_CM_01 & \\
\hline & & & & LOP_CCM & & OP_CM_25 & \\
\hline
\end{tabular}

\section{Case Study}

\subsection{Paper Positioning Devices}

Here, a paper positioning device used in printing presses is taken to illustrate the efficiency and capability of the domain reconstruction and mutation approach in expanding the function-form matching solution space and enhancing the creativity of the generated matched physical structures.

In the printing machine, the paper positioning device is used to position the paper laterally and longitudinally in the printing plane before the to-be-printed paper enters the printing device to start printing so that the paper and printing plate are ready in the correct positions to guarantee high-quality printing. Input suitable parameters in the functional decomposition module of the creative conceptual design platform according to the functional requirements of the paper positioning device, functional decomposition AND-OR tree is obtained [49], as shown in Fig.6a. The subfunctions in the tree are described using 11 basic operation actions. The temporal orders and spatial positions of these basic operational actions are shown in
Fig. 6b, c.

\subsection{Domain synthesis}

Input the following parameters: $m \_n G M a x, m \_n G M i n$, $m \_$nOMax, $m \_$nOMin, and $m \_$nSoMax in the domain synthesis module's dialog box. Here, $m \_n$ GMax and $m$ nGMin are the maximum and the minimum number of subfunctions that the functional schemes need to contain. Moreover, $m \_$nOMax and $m \_$nOMin are the maximum and minimum number of basic operation actions that the subsets contain. $m$ nSoMax is the maximum number of subsets with a single-base operation action. The domain synthesis module automatically generates functional schemes that can meet the functional decomposition requirements and the spatial and temporal order requirements among the subfunctions determined in Fig. $6 \mathrm{~b}$ with a set of basic operation actions. Domain synthesis can be divided into the following two parts.

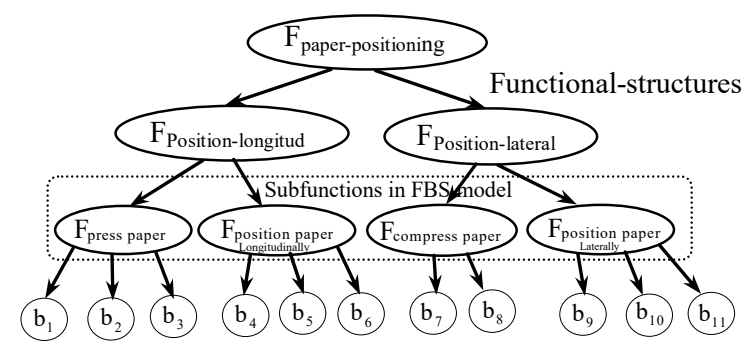

(a) Functional decomposition AND-OR tree

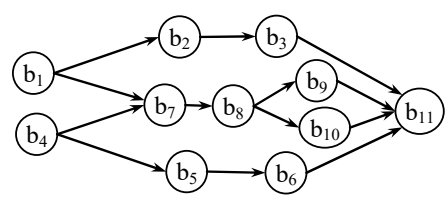

(b) Temporal orders

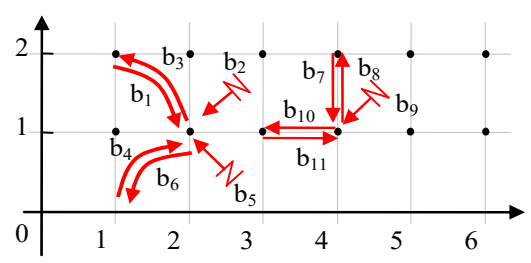

(c) Spatial positions

$\mathrm{b}_{1}$ : Swing the lateral pres tongue to working position, $C_{n c} ; \mathrm{b}_{2}$ : Stop the press tongue and press the paper, $S$ $\mathrm{b}_{3}$ : Avoids the paper for the press tongue, $C_{\mathrm{cr}} ; \mathrm{b}_{4}$ : Longitudinally position cardboard to working position, $C_{\mathrm{cr}}$

$\mathrm{b}_{5}$ : Stop the positioning cardboard and location paper, $S ; \mathrm{b}_{6}$ : Avoids the paper for the positioning cardboard, $C_{n}$ $\mathrm{b}_{7}$ : Press paper by the lateral positioning pressing head, $L ; \mathrm{b}_{8}$ : Stop pressing head and clamp paper, $S$

$\mathrm{b}_{7}$ : Press paper by the lateral positioning pressing head, $L ; \mathrm{b}_{8}$ : Stop pressing head and clamp paper, $S$
$\mathrm{~b}_{9}$ : Loosen pressing head and release paper, $L ; \quad \mathrm{b}_{10}$ : Pull paper by the lateral positioning pulling unit, $L$; $\mathrm{b}_{11}$ : Return of the lateral positioning pulling unit, $L$.

Figure 6 The functional decomposition for a paper positioning device

\subsubsection{Subsets partitioning}

For a group of parameters $\left\{m \_n G M a x, m \_n G M i n\right.$, $m \_$nOMax, $m \_$nOMin, $m \_$nSoMax $\}=\{4,1,9,2,1\}$, there are 314 partitioning schemes (that is, functional schemes) 
that can meet the top-level function requirements without considering the spatial and temporal order requirements among the partitioned subfunctions and among the basic operation actions in the partitioned subsets. For instance, Scheme 312, $\{[1,2]$, [3, 11, 9], [4, 5, 6], [7, 8, 10]\}, contains four subsets: $[1,2],[3,11,9],[4,5,6]$, and $[7,8$, 10]. In each subset, more than one basic operation action was contained. For example, subset $[3,11,9]$ contains three basic operation actions: $b_{3}, b_{11}$, and $b_{9}$, respectively.

\subsubsection{Domain synthesis}

First, the above-partitioned subsets should be synthesized in time and space orders based on the domain synthesis theory in quotient space [54]. The purpose of doing so is to guarantee that the subfunctions embodied by these partitioned subsets can meet the spatial and temporal order requirements among the basic operation actions. According to the domain synthesis theory, at the intersection of the time and spatial order synthesis spaces, the subfunctions embodied by the partitioned subsets can continuously and cooperatively realize the top-level functional requirements [47]. For the paper positioning device with the spatial and temporal order requirements among the basic operation actions, as shown in Fig. 6b, the number of functional schemes at the intersection of the time order synthesis space and the spatial order synthesis space is 167 . For instance, Scheme $18,\{[1,2,3]$, $[7,8,9],[4,5,6],[11,10]\}$ is one of the functional schemes.

\subsection{Domain Reconstruction}

The expansion of the solution space in the functional space and the physical space is implemented through domain reconstruction and mutation for the selected functional schemes obtained in the domain synthesis process. Select a functional scheme from them, such as Scheme 2, $\{[1,2,3,4,5,6],[7,8,9],[10,11]\}$, listed on the left Fig. 7a. The corresponding subsets of the basic operation actions are

$$
\left\{\left(b_{1}, b_{2}, b_{3}, b_{4}, b_{5}, b_{6}\right),\left(b_{7}, b_{8}, b_{9}\right),\left(b_{10}, b_{11}\right)\right\}
$$

However, no physical structure in the database can match this functional scheme based on the function-form direct mapping strategy, even with the heuristic functional-reasoning approach $[5,16,42]$, or the coevolution functional-reasoning approach [7, 20], and the hybrid approach of them [33]. In the function-form direct mapping FBS model, this functional scheme cannot be used to match the physical structure in the database. It will be deleted from the solution space if the domain reconstruction and mutation processes are not introduced, as in this study.

While no matched physical structure in the database can be figured out, the rule-based generating method [51] can be applied to create a new physical structure. Here, the absence of a matched physical structure in the database means that no matched physical structure can be sought out even though the rule-based generating method is adopted.

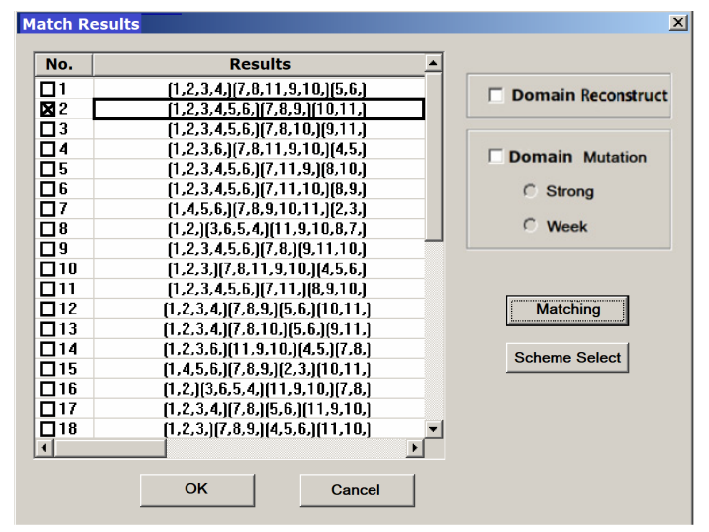

(a)

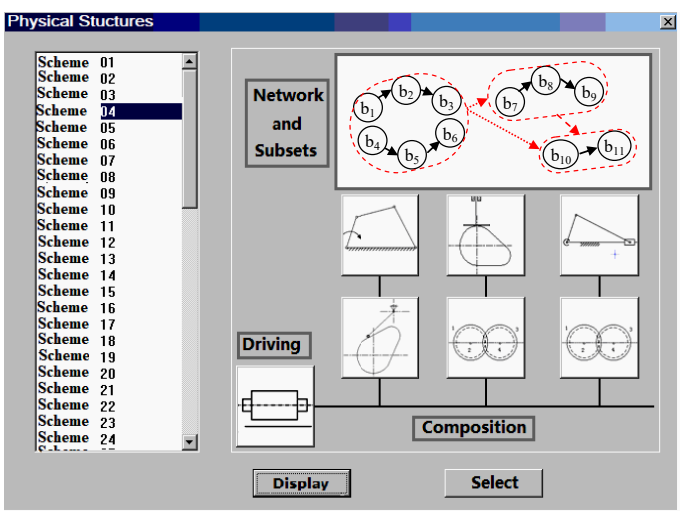

(b)

Figure 7 Domain reconstruction results related to the subset $\left\{\left(b_{1}, b_{2}, b_{3}, b_{4}, b_{5}, b_{6}\right),\left(b_{7}, b_{8}, b_{9}\right),\left(b_{10}, b_{11}\right)\right\}$

\subsubsection{Domain reconstruction}

However, while introducing the domain reconstruction process to the design problem for expanding the creative functional solution space, a total of 63 types of matched physical structures can be found related to the functional scheme $\left\{\left(b_{1}, b_{2}, b_{3}, b_{4}, b_{5}, b_{6}\right),\left(b_{7}, b_{8}, b_{9}\right),\left(b_{10}, b_{11}\right)\right\}$ if selecting single driving structures under the domain reconstruction menu term shown in Fig. 7a. In this functional scheme, the subset $\left(b_{7}, b_{8}, b_{9}\right)$ realizes the function of the press paper on the side gauge. The subset $\left(b_{10}, b_{11}\right)$ executes the drawing paper function on the side gauge and side positioning as a united subfunction. 
The subset $\left(b_{1}, b_{2}, b_{3}, b_{4}, b_{5}, b_{6}\right)$ implements two subfunctions: paper pressing and horizontal positioning of the front gauge. In one of the matched physical structures, such as Scheme 04, as shown in Fig. 7b, these two subfunctions, paper pressing and horizontal positioning of the front gauge, are embodied by a combined mechanism of a crank-rocker mechanism and an oscillating follower cam. In more detail, the function of paper pressing is realized by an oscillating follower cam with one primary function contributed by two basic operation actions $b_{1}$ and $b_{2}$ (that is, $C_{c r}$ and $C_{n c}$ ) and one auxiliary function contributed by one basic operation action $b_{3}(S)$. The crank-rocker mechanism supplies the subfunction of the horizontal positioning of the front gauge with its primary function contributed by $b_{4}$ and $b_{5}\left(C_{c r}\right.$ and $C_{n c}$ ) and an auxiliary function contributed by $b_{6}(S)$. The rocker in the crank-rocker mechanism can provide the one-dwell motion, $S\left(b_{6}\right)$, determined by its inherent characteristics when the specifically shaped coupling curve on the cam's follower drives the crank-rocker mechanism. It can be found that the combined mechanism is no more complicated than other matched mechanisms related to the subset $\left(b_{7}, b_{8}, b_{9}\right)$ or subset $\left(b_{10}, b_{11}\right)$.

Making full use of the intrinsic characteristics of mechanisms to dig out the ingenious combination of mechanisms with the most simplified compositions is essential for human experts to pursue excellent creative design results for mechanical systems. This case shows that the domain reconstruction method can effectively imitate human experts' creative design skills to realize ingenious combinations of mechanisms. Especially with computers' powerful reasoning mechanisms and their corresponding databases, it is possible to obtain creative design schemes with the platform proposed in this paper, which will be not inferior to the level of innovation of human experts. Moreover, when more basic operation actions are included in the subsets, the subfunctions may be realized by relatively simple matched physical structures. Thus, the novelty of the matched physical structures is improved significantly. Remarkably, while the multiple functional behaviors of the matched physical structures take part in the function-form matching process [46], it is practical and feasible to generate more simplified mechanical systems with few constituent building blocks to realize complex top-level functions. In other words, the domain reconstruction process for carrying out the functional synthesis presented in this paper can significantly improve the creativity of the matched physical structures, and expand the solution space to the creative solution space [13], for instance, the physical structures related to the function scheme (2): $\{[1$, $2,3,4,5,6],[7,8,9],[10,11]\}$.

\subsubsection{Domain mutation}

Under the domain mutation menu in Fig. 7a, two choices for strong and weak mutations are provided. Rules 3 and 4 in Section 3.1 are adopted in the mutation process while selecting the strong mutation. If a weak mutation is chosen, Rules 1 and 2 are adopted.

Here, we take a functional scheme, such as Scheme 18,

$$
\left\{\left(b_{1}, b_{2}, b_{3}\right),\left(b_{7}, b_{8}, b_{9}\right),\left(b_{4}, b_{5}, b_{6}\right),\left(b_{11}, b_{10}\right)\right\}
$$

obtained through the domain synthesis as an example to illustrate in detail. In this scheme, the subset of basic operation actions, $\left(b_{1}, b_{2}, b_{3}\right)$, realizes the paper-pressing function at the front gauge of the positioning mechanism. The subset $\left(b_{4}, b_{5}, b_{6}\right)$ completes the paper horizontal blocking positioning function at the front gauge. The subset $\left(b_{7}, b_{8}, b_{9}\right)$ realizes the paper-pressing function at the side gauge, and the subset $\left(b_{11}, b_{10}\right)$ realizes the drawing paper and lateral positioning function at the side gauge. When the strong mutation operation is adopted, and the multiple driving devices are chosen as the matched physical structures, 108 types of matched physical structures can be determined, as shown in Fig. 8a.

Similarly, for Scheme 18, a weak mutation operation is adopted. Namely, Rules 1 and 2 in Section 3.1 are taken as the mutation rules. When selecting multiple driving devices, a total of 135 groups matched physical structure schemes satisfying the functional requirements are determined, as shown in Fig. 8b on the left. The detailed construction for one physical structure, without losing generality selecting one of them, Scheme 13 is shown in Fig.8b in the lower right. Compared with the physical structure scheme in Fig. 8a, the physical structure scheme in Fig. $8 \mathrm{~b}$ is a mutating structure. The basic operation action performing the curve motion in the original scheme (Fig. 8a) changes to one performing linear line motion (Fig. 8b). Thus, novel and innovative physical structure schemes are generated through the mutation of basic operation actions. Moreover, the number of matched physical structures related to the top-level function has been significantly reduced. For instance, the physical structures are simplified from the seven constituent mechanisms (or building blocks) in Fig. 8a to the five constituent mechanisms in Fig. 8b. This case indicates that the domain mutation approach presented in this paper can expand the function-form mapping solution space and obtain more simplified physical structures, that is, with few constituent devices to realize complex top-level functions. 


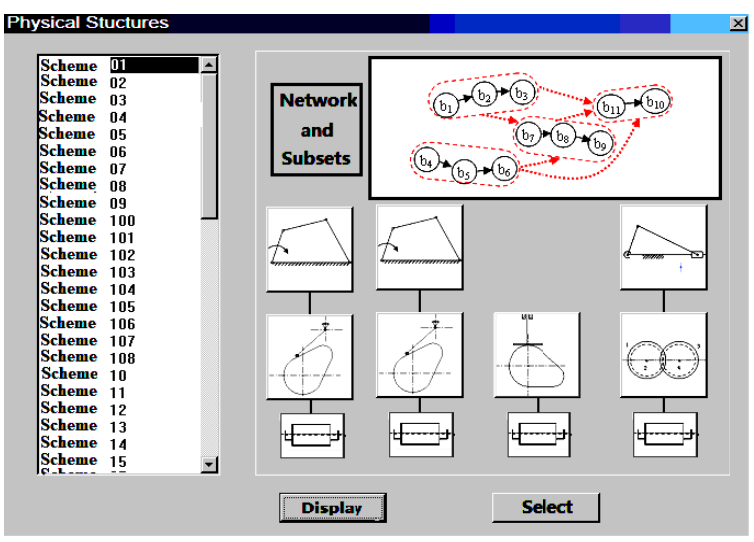

(a) Without introducing mutation process

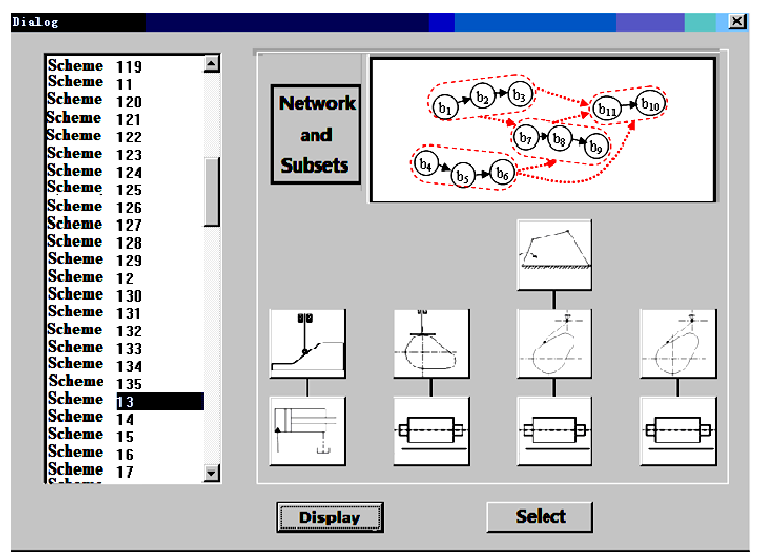

(b) Introducing mutation process

Figure 8 Matched physical structures related to scheme $\left\{\left(b_{1}, b_{2}, b_{3}\right),\left(b_{4}, b_{5}, b_{6}\right),\left(b_{7}, b_{8}, b_{9}\right),\left(b_{10}, b_{11}\right)\right\}$

\section{Discussion}

\subsection{Merits or advantages}

With fewer and simpler constituent physical structures to implement the top-level function of a design problem, the matched physical structures' creativity is undoubtedly improved. The computerized platform presented in this paper achieves this goal through the functional domain synthesis process, the domain reconstruction and mutation process on the one hand, and making the multiple behaviors of each building block participate in function-form matching on the other hand. The domain synthesis process is a bottom-up rule-based functional synthesis process. Different partitions will lead to generating different sub-functions and functional structures for the same design requirements. So it has a strong capability to introduce new design variables into the functional synthesis process and provides many functional hierarchies. The domain reconstruction and mutation processes introduced in this paper further expand the functional and physical solution space to the creative design space. Furthermore, by making each building block's multiple behaviors participate in function-form matching and enlarging the number of basic operation actions in the matching subfunctions, more simplified mechanical systems with few constituent devices can be figured out to implement the overall functional requirements.

The compatibility, time, and space continuity of the basic operation actions are described and recorded by the quotient topology attributes and structure attributes while setting up the functional decomposition and/or tree, as shown in Fig. 6a. These relationships among the basic operation actions in the form space are also well defined and described in constructing the database of common mechanisms, as listed in Table 2. Because the clustering approach [47] embedded in the domain reconstruction and mutation processes can guarantee that the synthesized subsets of basic operation actions perform well-organized and complete kinematic behaviors, and matched physical structures in the database embody these kinematic behaviors orderly and continuously [48], the obtained matched physical structures can realize the given top-level function organically and cooperatively.

The domain reconstruction and mutation methods presented in this paper belong to the rule-based generative approach $[25,36]$. They do not need AI-based reasoning knowledge to support the functional synthesis process. As a result, they can significantly expand the functional and physical solution spaces to the creative design spaces [13] to deal with non-routine creative conceptual design problems and synthesize the combinatorial mechanisms in composite and parallel connections, where, no direct or indirect knowledge to support the synthesis reasoning from the constituent mechanisms to their combination.

Furthermore, because the domain reconstruction and mutation rules are objectively defined by the compatibility of the kinematic behaviors of the basic operation actions, the temporal order and/or spatial position continuity, or the characteristics of basic operation actions, the major challenge of a lack of quantitative information to scale evaluating fitness [33] and the difficulty in determining objective grammar rules [38] for grammar-based design systems no longer exist here.

The characteristics with fewer and simpler constituent physical structures to meet and implement the functional requirements organically and synergistically determine that the schemes of physical structures figured out by the platform have prominent market competitive advantages in low manufacturing and maintenance costs, the long service life of the product, and high reliability. To our best 
knowledge, there is no such literature to deal with the problems of the behavioral reorganization and mutation of constituent artifacts for figuring out more innovative constituent physical structures to realize the overall functional requirements of design problems.

\subsection{Limitation and Further Work}

\subsubsection{Limitation}

Due to the lack of knowledge to support the functional synthesis of parallel and composite mechanisms, the function-based synthesis of parallel and composite mechanisms has become one of the biggest challenges in mechanism science and theory for over a century $[2,55]$. Especially, parallel and composite mechanisms have been widely used in daily life today, solving this puzzle is of significant merit. Although some methods to synthesize parallel and composite mechanisms based on topological characteristics of mechanisms were proposed [57, 58], they cannot deal with the trajectory-based synthesis problems, which are the core and critical synthesis problems of parallel and composite mechanisms to reflect human experts' ingenious combination of mechanisms fully. Currently, the trajectory-based synthesis problem of combined mechanisms has been solved by the platform. The other two kinds of synthesis problems: input-output synthesis and position synthesis, should be dealt with further, mainly focusing on defining the reconstruction and mutation rules related to different synthesis problems.

The high abstractness of mathematical description has two sides, both an advantage and a disadvantage. The high abstractness of comprehensive problems is conducive to expanding the functional solution space and facilitating computer recognition and processing. Maybe, the reader may have some difficulties in understanding. Nevertheless, for continuity of our previous research, the authors still expect to establish an abstract mathematical model based on the functional-based synthesis method and improve the abstraction and simplicity of the description of the methodology and platform to facilitate the popularization and transplantation of the platform in other application fields.

\subsubsection{Further work}

The creativity and innovation of the ideation is a crucial metric [59-62]. One of the most acknowledged for measuring ideation effectiveness is the four distinct metrics, quantity, quality, variety, and novelty, proposed by Shah et al. These four metrics corresponding to different levels of innovation can be effectively embodied and reflected by the platform. The explanation about this point of view will be given in detail in the following article.

Because the above four metrics for measuring the creativity of the outcome of the ideation is deduced based on the inquiry and survey of the experienced designers, it is necessary to establish an objective and systematic theory and platform for scaling and measuring the creativeness of the generated schemes according to the different levels and links of the automatic ideation provided by the platform. Then let it integrated with the platform, presented in this paper, in which the evaluation of the generated physical structures provided is based on the $3 \mathrm{D}$ automatic virtual simulation [50], through delegating novelty to the generated schemes in different ideation levels, the obtained physical structures will dominate in best physical structures and innovativeness uniformly.

Besides the included line and curve kinematic behaviors, the domain reconstruction and mutation rules for synthesizing mechanical systems should also include the input-output and position kinematic behaviors and the mathematical and physical operation functional elements, such as the physics-, logic-, and mathematic- based function units. Further, the rules supporting X-oriented manufacturing behaviors should also be considered.

\section{Conclusion}

The function-based synthesis of parallel and composite mechanisms is one of the biggest puzzles in mechanism science and theory for over a century due to the lack of knowledge to support mechanism combination from constituent mechanisms to their combination. This puzzle has been successfully solved by the platform proposed in this paper through imitating the creative design skills of human experts' ingenious combination of mechanisms to generate more innovative mechanical systems, which are not inferior to the level of innovation of human experts. Thus, it has great merit and significance in improving the creativity and market competitiveness of mechanical products.

The domain reconstruction and mutation in the platform are the rule-based synthesis processes. They do not need AI-based functional reasoning knowledge to support their reasoning processes. As a result, they can deal with the non-routine creative conceptual design problems and synthesize combined mechanisms in parallel and compound connections, where no knowledge supports the functional synthesis reasoning from the constituent mechanisms to their combinations. Because the domain reconstruction and mutation rules are objectively defined by the temporal order, the spatial 
position continuity, and the behavioral characteristics of the basic operation actions, as well as the compatibility of the kinematic behaviors contributed by the subset of basic operation actions, the challenge for the grammar-based design systems in deducing objective grammar rules that can search the global solution space and obtain the optimal innovative solutions robustly has been overcome.

The rule-based domain reconstruction and mutation processes have a strong capability to introduce new design variables into the functional synthesis process and expand the functional and physical solution space to the creative design space. Different domain synthesis rules will generate different sub-functions and functional structures for the same conceptual design problem. By making each constituent building block's multi-behaviors participate in function-form matching, the platform can obtain more simplified physical structures with fewer and simpler constituent devices to realize the overall functional requirements cooperatively and organically. Doubtlessly, a simple embodied mechanical system has prominent market competitive advantages over a complex one related to the same functional requirements.

\section{Funding}

Supported by National Natural Science Foundation of China (Grant No. 51775557, 51375496, 50875238, and 51575530).

\section{Availability of data and materials}

The datasets supporting the conclusions of this article are included within the article.

\section{Authors' contributions}

The author' contributions are as follows: Dr. Yu-Tong Li was in charge of the whole calculation and programming; Prof. Yu-Xin Wang was in charge of the whole technical solutions and wrote the manuscript.

\section{Competing interests}

The authors declare no competing financial interests.

\section{Consent for publication}

Not applicable

\section{Ethics approval and consent to participate}

Not applicable

\section{References}

[1] Hamel G. Strategy innovation and the quest for value. Sloan Manage Rev, 1998, Winter, 7-14.

[2] Zou H J, Liang Q H. Establishing distinctive theories and methods of design science rooted in Chinese traditional culture. Front Mech Eng, 2020, 15 (3): 430-437.

[3] Li J, Li Y. Research and application of computer aided design system for product innovation. J Comp Meth Sci Eng, 2019
19(S1): S41-S46.

[4] Sarkar P, Chakrabarti A. Ideas generated in conceptual design and their effects on creativity. Res Eng Design, 2014, 25: 185-201.

[5] Yilmaz S, Seifert C M, Gonzalez R. Cognitive heuristics in design: Instructional strategies to increase creativity in idea generation. Artif Intell Eng Des Anal Manuf, 2010, 24, 335-355.

[6] Tan R H, Liu W, Cao G Z, Shi Y. Creative design inspired by biological knowledge: Technologies and methods. Front Mech Eng, 2019, 14 (1): 1-14

[7] He B, Feng P E. Guiding conceptual design through functional space exploration. Artif Intell Eng Des Anal Manuf, 2013, 66:1999-2011.

[8] Müller J R, Isaksson O, Landahl J, Raja V, Panarotto M, et al. Enhanced function-means modeling supporting design space exploration. Artif Intell Eng Des Anal Manuf, 2019, 33(4):502-516.

[9] Chakrabarti A, Bligh T P. A scheme for functional reasoning in conceptual design. Des Studies. 2001, 22: 493-517.

[10] Pahl G, Beitz W. Engineering design: A systematic approach, 2nd ed. London: Springer, 1996.

[11] Tor S B, Britton G A, Zhang W Y, Deng Y M. Guiding functional design of mechanical products through rule-based causal behavioural reasoning. Int J Prod Res, 2002, 40(3):667-682.

[12] Roy U, Pramanik N, Sudarsan R, Sriram R D. Function-to-form mapping: model, representation and applications in design synthesis, CAD Comp Aided Des, 2001, 33(10): 699-719.

[13] Gero J S. Routine and non-routine design: a prototype-based approach. In: Inter conf exp syst eng appl. Wuhan, China, 1989, 369-371.

[14] Wook K L, Greer J. Function-based synthesis methods in engineering design: State-of-the-art, methods analysis, and visions for the future. Formal engineering design synthesis. Cambridge: Cambridge University Press, 2001, 170-227.

[15] Erden M S, Komoto H, Beek V T J, D'amelio V, Echavarria E, Tomiyama T. A review of function modeling: Approaches and applications. Artif Intell Eng Des Anal Manuf, 2008, 22: 147-169.

[16] Zhang M, Li G X, Gong J Z, Wu B Z. A hierarchical functional solving framework with hybrid mappings for supporting the design process in the conceptual phase. Proc IMechE Part B: J Eng Manuf, 2012, 226(8):1401-1415.

[17] Lin Y, Liu Y S, Sun Z F, Cao Y L, Qamar A. A hybrid approach for the automation of functional decomposition in conceptual design. $J$ Eng Des, 2016, 27(4-6): 333-360.

[18] Guo X, Liu Y, Zhao W, Wang J, Chen L. Supporting resilient conceptual design using functional decomposition and conflict resolution. Adv Eng Informatics, 2021, 48(4): 101262.

[19] Jia X, Jiang C, Fu C, et al. Uncertainty propagation analysis by an extended sparse grid technique. Front Mech Eng, 2019, 14(1): 33-46.

[20] Hui Q, Li Y, Tao Y, Liu H W. Triple-Helix Structured Model Based on Problem-Knowledge-Solution Co-evolution for Innovative Product Design Process. Chinese J Mech Eng (English Edition), 2020, 33(6): 49-63

[21] Pal U, Liu Y C, Chakrabarti A. Evaluating FuncSION: A software for automated synthesis of design solutions for stimulating ideation during mechanical conceptual design. Artif Intell Eng Des Anal Manuf, 2014, 28 (3): 209 - 226

[22] Chiou S, Kota S. Automated conceptual design of mechanisms. Mech Mach Theory, 1999, 34(3): 467-495.

[23] Bolognini F, Seshia A, Shea K. Exploring the application of multi-domain simulation-based computational synthesis methods in MEMS design. In Proceedings of 16th Int Conf Eng Des, 2007, Paris, France, 81-82.

[24] Campbell M I, Cagan J, Kotovsky K. An agent-based approach to conceptual design in a dynamic environment. Res Eng Des, 1999, 11(3): $172-192$

[25] Chakrabarti A, Shea K, Stone R, Cagan J, Campbell M, Hernandez $\mathrm{N} \mathrm{V}$, Wood K L. Computer-based design synthesis research: an overview, J Com Info Sci Eng, 2011, 11(6): 021003-1-10.

[26] Fiorineschi L, Rotini F, Rissone P. A new conceptual design 
approach for overcoming the flaws of functional decomposition and morphology. J Eng Des, 2016, 27(7): 438-468.

[27] Fiorineschi L. Abstraction framework to support students in learning creative conceptual design, J Eng Des Tech, 2018, 16(4) : 616-636.

[28] Antonsson E K, Cagan J, eds. Formal engineering design synthesis. Engineering shape grammars. Cambridge: Cambridge University Press, 2001, 65-92

[29] Schmidt L C, Shetty H, Chase S C. A Graph Grammar Approach for Structure Synthesis of Mechanisms. ASME J Mech Des, 2000, 122(4): 371-376.

[30] Helms B, Shea K. Computational Synthesis of Product Architectures Based on Object-Oriented Graph Grammars. ASME J Mech Des, 2012,134(2): 021008 .

[31] Han L, Liu G, Yang X H, Han B. A Computational Synthesis Approach of Mechanical Conceptual Design Based on Graph Theory and Polynomial Operation. Chinese J Mech Eng (English Edition), 2020, 33(1): 60-74.

[32] Ostrosi E, Salima T B, Lianda H, Didier K. A grammar approach for combining product configuration modeling with behavioural aspects and simulations. Int $J$ Adv Manuf Technol, 2012, 60: 29-54.

[33] Jin Y, Li W. Design Concept Generation: A Hierarchical Coevolutionary Approach. J Mech Des, 2007, 129(10): 1012-1022.

[34] Albers A, Rovira N L, Aguayo H, Maier T. Optimization with genetic algorithms and splines as a way for computer aided innovation. IFIP Adv Inf Com Tech, 2008, 277:7-18.

[35] Li P, Ren Y Z,Yan Y, Wang G X. Conceptual design method driven by product genes. Proc IMech Eng Part B: J Eng Manuf, 2020, 234(3): 463-478.

[36] Robert F, Woodbury, eds. Modeling creativity and knowledgebased creative design: A genetic approach to creative design. London: Lawrence Erlbaum Associates Inc., 1993.

[37] Li B, Chen L, Huang Z, Zhong Y. Product configuration optimization using a multi objective genetic algorithm. Int $J A d v$ Manuf Technol, 2007, 30(1-2): 20-29.

[38] Zhang J, Du H, Xue D, Gu P. Robust design approach to the minimization of functional performance variations of products and systems. Front Mech Eng, 2021, 16:379-392.

[39] Vermaas P E. On the formal impossibility of analyzing subfunctions as parts of functions in design methodology. Res Eng Des, 2013, 24 (1): 19-32.

[40] Dingmar V E, Daniel A M. Functional Decomposition in Engineering: A Survey. In Proceedings ASME 2007 IDETC/CIE, New York: ASME, Las Vegas, USA, 2007.

[41] Eck D V, Mcadams D A, Vermaas, P E. Functional decomposition in engineering: A Survey. In Proceedings ASME Inter Des Eng Tech Conf Comp Infor Eng Conf, 2008: 227-236.

[42] Xue D Y, Imaniyan D. An Integrated Framework for Optimal Design of Complex Mechanical Products. J Com Infor Sci Eng, 2021, 21(4): 1-32.

[43] Welch R V, Dixon J R. Guiding conceptual design through behavioral reasoning. Res in Eng Des, 1994, 6(3): 169-188.

[44] Chen, B. Conceptual design synthesis based on series-parallel functional unit structure. J Eng Des, 2018, 29(3): 87-130.

[45] Umeda Y, Kondoh S, Shimomura, Y, Tomiyama T. Development of Design Methodology for Upgradable Products based on Function-Behaviour-State Modelling. Artif Intell Eng Des Anal Manuf, 2005, 19: 161-182

[46] Li Y T, Wang Y X, Duffy A H. Computer-based creativity enhanced the conceptual design model for the non-routine design of mechanical systems. Chinese J Mech Eng (English Edition), 2014, 27(6): 1083-1098

[47] Li Y T, Wang Y X. A Bottom-up Functional Domain Synthesis Approach for Creative Conceptual Design. The Sixth International Conference on Design Creativity (ICDC2020). Oulu, Finland, August 26th $-28^{\text {th }}, 2020$

[48] Li Y T, Wang Y X. The innovation of matching structures through clustering and reconstructing basic operation actions in the form layer. In Proceedings ASME Inter Des Eng Tech Conf Comp Infor
Eng Conf, DETC2017-67307, 2017.

[49] Li Y T, Wang Y X. A mathematical functional decomposition approach through granularity partition process in quotient space. In Proceedings ASME Inter Mech Eng Cong Expo, IMECE2018 $-86217,2018$.

[50] Li Y T, Wang Y X. A systematic rapid creative design framework for complex mechanical systems from conceptual design to $3 \mathrm{D}$ virtual simulation. In Proceedings ASME Inter Des Eng Tech Conf Comp Infor Eng Conf, DETC2019-98150, 2019.

[51] Li Y T, Wang Y X. A bottom-up function-based synthesis approach for complex mechanisms based on clustering rules. Mech based Des Struc Mach, 2020, 47 (5):555-56.

[52] Wang Y X, Mao X H, Li T Y. Key techniques in function-form bidirectional creativity quotient space model. J Zhejiang Univ. (Eng Sci), 2010, 44(9):1643-1653.

[53] Deng Y M, Zhu Y W. Function to structure/material mappings for conceptual design synthesis and their supportive strategies. Int $J$ Adv Manuf Technol, 2009, 44:1063-1072.

[54] Zhang L, Zhang B. A quotient space approximation model of multi-resolution signal analysis. J Comp Sci Tech, 2005, 20(1): 90-94.

[55] Zou H J, Liang Q H, Zhang Q. Theory and method of mechanism system design. Front Mech Eng, 2010, 5 (4): 399-411.

[56] Pan Y, Gao F, et al. Position model computational complexity of walking robot with different parallel leg mechanism topology patterns. Mech Mach Theory, 2017, 107:324-337.

[57] Huang L, Gao Y, Feng Q, Tang S, Wang D L. Configuration Selection for Reconfigurable Manufacturing Systems by Means of Characteristic State Space. Chinese J Mech Eng, 2011, 24(1):23-32.

[58] Tang L. An approach to function identification in automated conceptual design of mechanism systems. Res Eng Design, 2008,19: $151-159$.

[59] Shah J J, Vargas-Hernandez N, Smith S M. Metrics for measuring ideation effectiveness. Des Stud, 2003, 24(2): 111-134.

[60] Sarkar P, Chakrabarti A. Assessing design creativity, Des Stud, 2011, 32 (4):348-383.

[61] Fiorineschi L, Frillici F S, Rotini F. A-Posteriori Novelty Assessments for Sequential Design Sessions. In: Inter Des Conf Des, 2018, 2018: 1079-1090.

[62] Hay L, Duffy A H B, Grealy M, Tahsiri M, McTeague C, Vuletic T. A novel systematic approach for analyzing exploratory design ideation. J Eng Des. 2019, 31(3):127-149.

\section{Biographical notes}

Yu-Tong LI is currently an associate professor of China University of Petroleum Huadong. She received her Dr. degree from Zhejiang University (2010) and primary engaging in creative design theory of the conceptual design process based on the quotient space theory; singularity avoidance of the parallel mechanism, especially the vector driving mechanism of the axisymmetric vectoring exhaust nozzle. E-mail: lyt_b@163.com

Yu-Xin WANG, born in 1964, is currently a professor in China University of Petroleum Huadong. He was a professor at Tianjin University (1996-2000), during this period, he won the first-class prize (research) for young teachers from Fok Yingdong Education Foundation with a higher than 18 H-index; Tongji University (2001-2007); and Zhejiang University (2008-2011). His primary interest is creative design theory and mathematical automation model based on the quotient space theory, gordian techniques in the axisymmetric vectoring exhaust nozzle (AVEN).

E-mail: strath_tj@hotmail.com 